Sonderdruck aus

\title{
Archiv der Pharmazie
}

301. Band, Heft 1, Seite 50-58

Verlag Chemie, G mbH, Weinheim / Bergstr.

\author{
W. Wiegrebe, U. Krüger, H. Reinhart und L. Faber \\ Überführung von 1-Benzylisochinolinen in Berbinium-Salze
}

6. Mitt.: Synthesen und NMR-Spektren von Coralyn-Derivaten 


\author{
W. Wiegrebe, U. Krüger, H. Reinhart und L. Faber
}

\title{
Überführung von 1-Benzylisochinolinen in Berbinium-Salze
}

\section{Mitt.: Synthesen und NMR-Spektren von Coralyn-Derivaten*) **)}

Aus dem Institut für Pharmazeutische Technologie der Technischen Hochschule Braunschweig und aus dem Institut für Molekulare Biologie, Biochemie u. Biophysik, Stöckheim b. Braunschweig

(Eingegangen am 21. Juli 1967)

Der Abbau des Coralyns zum Norcoralyn über die Zwischenstufe der Papaverin-6'. carbonsäure und eine Synthese des Norcoralyns durch Vilsmeier-Reaktion aus Papaverin werden beschrieben. - Im Gegensatz zu Literaturangaben konnten wir 9-Methylpapaverin in ein Coralyn-Derivat überführen. - Die NMR-Spektren einiger Coralyn-Abkömmlinge werden besprochen.
The degradation of coralyn to norcoralyn via $6^{\prime}$-papaverinecarboxylic acid and a synthesis of norcoralyn by Vilsmeier-reaction of papaverine are described. - Contrary to the literature we were able to transform 9-methylpapaverine to a coralyn-derivative. - The NMR-spectra of several coralynderivatives are discussed.

Seit etwa 50 Jahren ist das 2,3,10,11-Tetramethoxy-8-methyl-dibenzo[a,g]-chinolizinium-Ion bekannt, das von Schneider et al. ${ }^{1}$ ) erstmals hergestellt und ,Coralyn“" $\left(\mathrm{I}, \mathrm{R}^{\prime}=\mathrm{CH}_{3}\right)$ genannt wurde. Coralyn (I, $\mathrm{R}^{\prime}=\mathrm{CH}_{3}$ ) entsteht aus Papaverin (II) durch Reaktion mit Acylkationen. Läßt man nicht Acetylchlorid bzw. Acetanhydrid, sondern entsprechende Derivate anderer Säuren auf II einwirken, so erhält man Coralyn-Homologe, in denen die 8-Methyl-Gruppe durch andere Alkyle bzw. durch Arylreste ersetzt ist $\left.\left.{ }^{1}\right)^{2}\right)^{3}$ ).

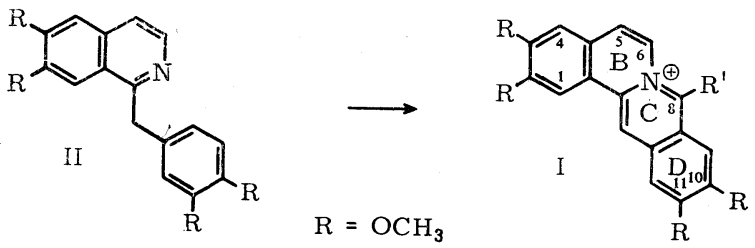

Das am C-8 u nubstituierte Coralyn-Analoge, das sog. ,Norcoralyn“ $\left(I, R^{\prime}=H\right)$, läßt sich dagegen durch Gattermann-Synthese, auch in den variierten Formen nach Koch und Adams, nicht herstellen $\left.{ }^{3}\right)$. Die erste Synthese des Norcoralyns $\left(\mathrm{I}, \mathrm{R}^{\prime}=\mathrm{H}\right)$

*) 5. Mitt.: W. Wiegrebe, D. Sasse und E. Roesel, Arch. Pharmaz. 301, 33 (1968).

**) Herrn Prof- Dr. H.W. Bersch zum 65. Geburtstag gewidmet.

1) W. Schneider et al., Ber. dtsch. chem. Ges. 53, 1459 (1920); 54, 2021 (1921)

2) W. Awe, Pharmaz. Zentralhalle Deutschland 77, 157 (1936).

3) H. Halpaap, Diss. Braunschweig 1950, S. 32. 
gelang Bradsher et al. ${ }^{4}$ ), die das Oxim des 1-Formyl-6,7-dimethoxy-N-veratrylisochinolinium-Salzes mit Säure zu $I, R^{\prime}=H$ cyclisierten.

Das Ziel unserer Arbeit war die Umwandlung des Coralyns in Norcoralyn. Behandelt man Coralyn mit Lauge, so entsteht $6^{\prime}$-Acetyl-papaverin (III) ${ }^{1}$ ), das mit Hypobromit in Papaverin-6'-carbonsäure (IV) überführt und als Hydrochlorid isoliert wurde.

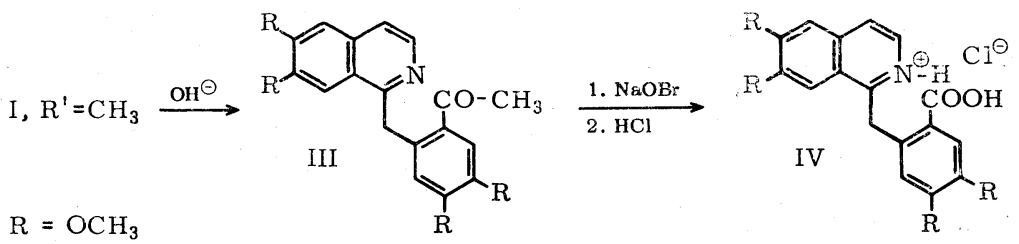

Durch Reduktion von IV-Hydrochlorid mit Lithiumaluminiumhydrid in Pyridin ${ }^{5}$ ) erhielten wir in schlechten Ausbeuten 6'-Hydroxymethyl-papaverin (V) und Norcoralyn $\left(\mathrm{I}, \mathrm{R}^{\prime}=\mathrm{H}\right)$, das als Chlorid isoliert und durch sein UV-Spektrum charakterisiert wurde. Bei der Reduktion dieser Verbindung entstand Norcoralydin ${ }^{6}$ ).
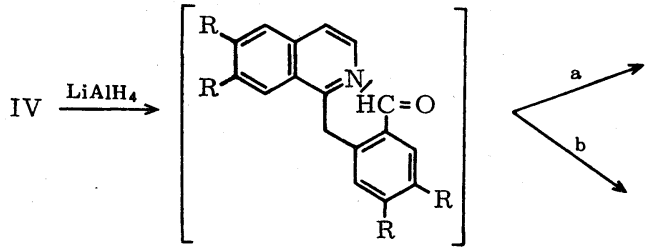<smiles>[R]c1cc(CO)c(Cc2nccc3cc([R])c([R])cc23)cc1[R]</smiles>

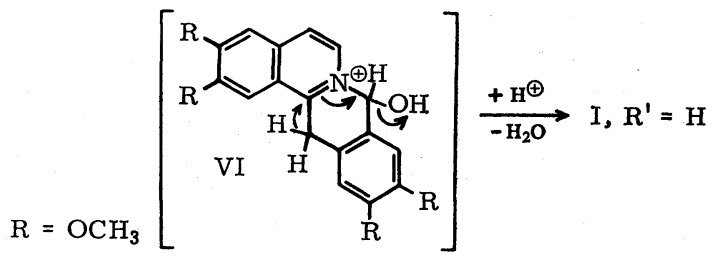

Die Norcoralyn $\left(\mathrm{I}, \mathrm{R}^{\prime}=\mathrm{H}\right)$-Bildung war aus folgendem Grund zu erwarten: Die Reduktion einer Carbonsäure zum Carbinol durchläuft die Oxydationsstufe des Aldehyds ${ }^{7}$ ). In unserem Fall sollte die nucleophile Substitution der AldehydCarbonyl-Gruppe (bzw. des Åquivalentes) durch ein weiteres Hydrid-Ion (a) mit dem nucleophilen Angriff durch den Isochinolin-Stickstoff (b) zum Aminoalkoholat-

4) C. K. Bradsher und N.L. Dutta, J. org. Chemistry 26, 2231 (1961).

5) Zur Arbeitstechnik s. P. T. Lansbury, J. Amer. chem. Soc. 83, 429, 3537 (1961).

6) A. Pictet und T. Q. Chou, Ber. dtsch. chem. Ges. 49, 370 (1916).

- $\left.{ }^{7}\right)$ J. D. Roberts und M. C. Caserio, Basic Principles of Organic Chemistry, W. A. Benjamin Inc., New York 1964, S. 522/523. 
Ion konkurrieren. Bei der sauren Aufarbeitung spaltet der freie Aminoalkohol VI Wasser ab, und es entsteht Norcoralyn.

Über die Struktur der Zwischenprodukte auf der Aldehydstufe bei der Reduktion von Carbonsäuren ist nichts Sicheres bekannt ${ }^{7}$ ). Sicherlich liegt nicht die freie Aldehydgruppe vor, die hier aus Gründen der Übersichtlichkeit formuliert wurde.

Da die direkte Reduktion des Carbonsäure-Hydrochlorids (IV) eine unbefriedigende Ausbeute gab, wurde IV über das Methylester-Hydrochlorid in den freien IV-Methylester umgewandelt, der mit Lithiumaluminiumhydrid zu V reduziert wurde. Der Versuch, V mit aktivem Braunstein selektiv zum Aldehyd zu oxydieren, der durch Säureeinwirkung zu $\mathrm{I}, \mathrm{R}^{\prime}=\mathrm{H}$ cyclisiert hätte, mißlang. Wir erhielten eine schwefelgelbe, N-haltige Substanz, die zwar blaugrün, aber nicht gelbgrün wie Coralyn $\left(\mathrm{I}, \mathrm{R}^{\prime}=\mathrm{CH}_{3}\right)$ fluoreszierte. Beim Behandeln mit $\mathrm{n} \mathrm{HCl}$ bildete diese Substanz kein Salz, und da sie im IR-Spektrum eine Bande bei 1650/cm aufwies, wurde sie als Neutralstoff mit einer Lactamgruppierung (VII) angesprochen.

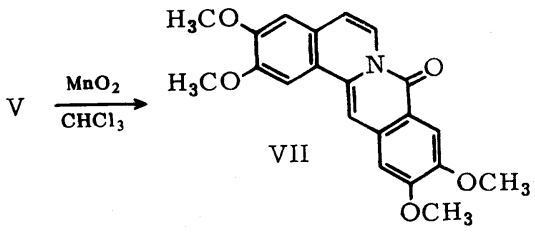

Zur Sicherung der Struktur VII wurde das Massenspektrum aufgenommen*): es zeigte das Signal des Molekülions bei der für VII berechneten Massenzahl (365). Im Massenspektrometer verlor das Molekül eine Methylgruppe (Signal bei M-15 m/e). Bei der Reduktion mit Zink und Säure entstanden aus VII zwei Verbindungen, die im DC sehr nahe beieinander lagen, und von denen eine mit Norcoralydin 6 ) identisch war. Das beweist, daß VII das formulierte Ringsystem besitzt. Das Lactam VII ist wahrscheinlich folgendermaßen entstanden: das Carbinol V wurde zum entsprechenden Aldehyd oxydiert, der durch die bei der direkten Carbonsäure-Reduktion (s. o.) beschriebene nucleophile Substitution durch das Isochinolin- $\mathrm{N}$ in den ringgeschlossenen Aminoalkohol VI überging. Da wir im neutralen Milieu arbeiteten, und die Wasserabspaltung aus VI protonenkatalysiert ist, konnte VI zu VII weiteroxydiert werden.

Die Entstehung von VII stützt die Annahme, daß die Umsetzung der $6^{\prime}$ Acyl-1-benzylisochinoline zu entsprechenden Coralyn-Derivaten über die Zwischenstufe eines Aminoalkohols (analog VI) verläuft.

Kürzlich berichteten S. Hauptmann und A. Blakovits $\left.{ }^{8}\right)$ über eine vergleichbare Reaktion: sie fanden, daß bei der $\mathrm{MnO}_{2}$-Oxydation von 1,2-(Bishydroxymethyl)-benzol Phthalid entsteht. Die Autoren folgern, daß als Zwischenprodukt 2-Hydroxymethyl-benzaldehyd entsteht, der als Halbacetal weiteroxydiert wird.

*) Wir danken Herrn Dozent Dr. H. Budzikiewicz verbindlich für die Aufnahme und Interpretation des Massenspektrums_von VII.

8) Z. Chemie 6,466 (1966). 
Wir zogen aus der Lactambildung bei der $\mathrm{MnO}_{2}$-Oxydation in neutraler Lösung den Schluß, daß in einem neuen Experiment die Reaktionsbedingungen so zu wählen sind, daß der entstehende Aminoalkohol VI sehr schnell durch Säure zu $\mathbf{I}, \mathbf{R}^{\prime}=\mathbf{H}$ dehydratisiert wird. Bei der Oxydation des Carbinols V mit Dichromat in 50proz. Essigsäure fiel bei Raumtemp. nach ${ }^{1} / 2$ Std. ein kristallines Produkt aus, das aus Methanol mehrfach umkristallisiert wurde. Die Elementaranalyse ergab einen abnorm hohen ,,unverbrennlichen Rückstand“", mit Diphenylcarbazid zeigte die Substanz eine starke Violett-Färbung, so daß wir schließen konnten, daß das o. a. Kristallisat das Hydrogenchromat des Norcoralyns $\left(I, R^{\prime}=H\right)$ darstellte. Das NMR-Spektrum dieses Salzes zeigte fast keine Auflösung und sprach dafür, daß die Substanz paramagnetische Eigenschaften besitzt bzw. mit einem paramagnetischen Stoff verunreinigt war. Die Linienverbreiterung im NMR-Spektrum erklären wir damit, daß durch das Umkristallisieren in der Hitze Spuren von Cr(III)-Verbindungen entstanden waren. Bei der Reduktion des Norcoralyn-Hydrogenchromats entstand eine apfelgrüne Lösung, aus der wir Norcoralydin-Hydrochlorid $^{6}$ ) gewinnen konnten, das mit authentischer Substanz ${ }^{6}$ ) im Mischschmp. keine Depression zeigte und im IR-Spektrum deckungsgleich war. Wir kristallisierten daraufhin das Norcoralyn-Hydrogenchromat mehrmals aus salzsaurem Methanol um und erhielten Norcoralyn-Chlorid.

Die Synthese des Norcoralyns nach Bradsher ${ }^{4}$ ) und unsere Umwandlung des Coralyns in Norcoralyn sind nicht geeignet, Norcoralyn $\left(I, R^{\prime}=H\right)$ schnell und in größerer Menge herzustellen. Die Umsetzungsversuche von Papaverin (II) mit „Formylchlorid“" nach Gattermann ${ }^{3}$ ) schlugen fehl, da die für diese Reaktion notwendigen Lewis-Säuren (z. B. $\mathrm{AlCl}_{3}$ ) mit Benzylisochinolinen praktisch unlösliche Additionsverbindungen geben ${ }^{9}$ ). Wir umgingen diese Schwierigkeit, indem wir II mit Phosphoroxychlorid und Dimethylformamid nach Vilsmeier umsetzten und dabei schmelzpunktreines Norcoralynchlorid in 59proz. Ausbeute erhielten.

Unsere Arbeiten, die sich mit der Überführung von 1-Benzylisochinolinen in Berbinium-Salze beschäftigten, gehen von der Frage aus, in wie weit die CoralynReaktion für Papaverin spezifisch ist. W. Awe, H. Halpaap und O. Hertel ${ }^{10}$ ) haben in umfangreichen Arbeiten die Voraussetzungen erforscht, die notwendig sind, um
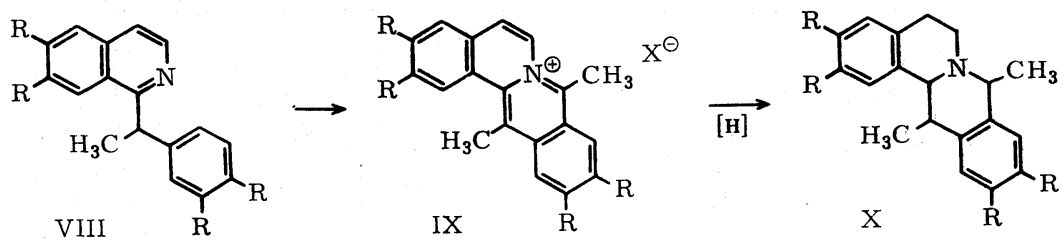

$$
\mathrm{R}=\mathrm{OCH}_{3}
$$

${ }^{9}$ W. Wiegrebe, Arch. Pharmaz. 300, 330 (1967).

10) Arzneimittel-Forsch. 10, 936 (1960). 
ein Benzylisochinolin vom Typ des Papaverins in ein Coralyn-Derivat überführen zu können. Diese Autoren berichten, daß 9-Methylpapaverin (VIII) sich nicht zu einem Coralyn-Analogen umsetzen ließe. Wir sahen für dieses Verhalten keine theoretische Begründung, wiederholten das Experiment und isolierten 13-Methylcoralyn (IX), das zu zwei isomeren - vermutlich diastereomeren - 13-Methylcoralydinen (X, Racemat I und II) reduziert wurde.

\section{NMR-spektroskopische Untersuchungen; Tabelle 1}

Lage der Signale in den $100 \mathrm{MHz}-\mathrm{NMR}$-Spektren von Norcoralyn $\left(I, R^{\prime}=H\right)$, Coralyn (I, $\mathrm{R}^{\prime}=\mathrm{CH}_{3}$ ) und 13-Methyl-coralyn (IX); chemische Verschiebung in $\tau(\mathrm{ppm})$. $\mathrm{CF}_{3} \mathrm{COOD}$ als Lösungsmittel, TMS als innerer Standard

\begin{tabular}{|c|c|c|c|c|c|c|c|c|c|c|}
\hline & \multirow{2}{*}{$\begin{array}{l}\text { Methylgruppen } \\
\text { in 8- und } \\
\text { 13-Stellung }\end{array}$} & \multirow{2}{*}{$\begin{array}{l}\text { Methoxygruppen in } \\
2-, 3-, 10-\text { und } \\
11-\text { Stellung }\end{array}$} & \multicolumn{8}{|c|}{ Aromatische Protonen } \\
\hline & & & 1 & 4 & 5 & 6 & 8 & 9 & 12 & 13 \\
\hline Norcoralyn & - & $\begin{array}{llll}5,74 & 5,82 & 5,80 & 5,95\end{array}$ & 1,80 & 2,43 & 2,20 & 1,50 & 0,48 & 2,41 & 2,54 & 0,82 \\
\hline Coralyn & 6,55 & $5,74 \quad 5,82 \quad 5,73$ & 1,73 & 2,38 & 2,08 & 1,31 & - & 2,24 & 2,50 & 0,77 \\
\hline coralyn & 6,63 & $\begin{array}{llll}5,76 & 5,81 & 5,87\end{array}$ & 2,04 & 2,37 & 2,23 & 1,53 & - & 2,27 & 2,56 & - \\
\hline
\end{tabular}

Bis auf die Signale der o-ständigen Protonen an den C-Atomen 5 und 6, die zu Dubletts mit $J_{56}=7,5 \mathrm{~Hz}$ aufspalten, treten die Signale aller aromatischen Protonen als Singuletts auf [ein Ausschnitt aus dem 100-MHz-NMR-Spektrum des Coralyns ist in der 5. Mitt. dieser Publikationsreihe abgebildet]. Die Integralkurve stimmt mit der Anzahl der zugeordneten Protonen überein. Das beim Norcoralyn im Vergleich zum Coralyn hinzukommende Proton am C-8 ist $\alpha$-ständig zum N und liegt in der Mitte des durch die Ringe B, C und D gebildeten, linear annelierten Systems. Für dieses Proton ist ein stark zu niedrigen Feldstärken hin verschobenes Signal zu erwarten, das bei $\tau=0,48 \mathrm{ppm}$ vor der Gruppe der dem Coralyn analogen Signale gefunden wird.

Dem Proton am C-13 wurde das, vom Bereich niedriger Feldstärke her gesehen, nächst folgende Signal bei $\tau=0,82 \mathrm{ppm}$ (Norcoralyn $\mathrm{I}, \mathrm{R}^{\prime}=\mathrm{H}$ ) bzw. $\tau=0.77$ (Coralyn $\mathrm{I}$, $\mathbf{R}^{\prime}=\mathrm{CH}_{3}$ ) zugeordnet. Für dieses Proton ist ebenfalls eine zu niedrigem Feld hin verschobene Lage zu erwarten; es ist $\mathrm{m}$-ständig zum $\mathrm{N}$ angeordnet und fast halbkreisförmig von aromatischen Ringen umgeben, deren Ringströme eine starke paramagnetische Verschiebung bewirken. Im Spektrum des 13-Methyl-coralyns (IX) fehlt dieses Signal.

Trägt man die Differenzen der Signallagen des Coralyns $\left(I, R^{\prime}=\mathrm{CH}_{3}\right) \mathrm{zu}$ denen des Norcoralyns ( $I, R^{\prime}=\mathrm{H}$ ) auf (Abb. 1), so ist eine zum $N$ hin zunehmende „downfield“-

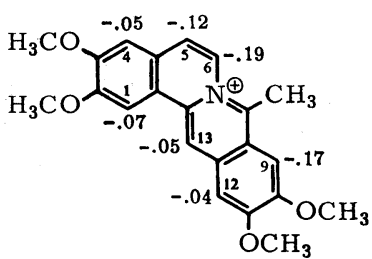

Abb. 1. Verschiebung der Coralyn-

Signale im Vergleich zum Norcoralyn

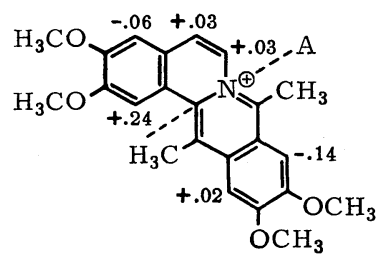

Abb. 2. Verschiebung der 13-Methylcoralyn-Signale im Vergleich zum Norcoralyn 
Verschiebung der Coralyn-Signale zu erkennen. Diese sich erwartungsgemäß abstufende Verschiebung werten wir als Hinweis darauf, daß die Signale richtig zugeordnet wurden.

In Abb. 2 sind die entsprechenden Differenzen in den Signallagen zwischen 13-Methylcoralyn (IX) und Norcoralyn eingezeichnet. Der Einfluß der C-13-Methylgruppe in IX auf das Proton am C-1 bewirkt wahrscheinlich eine Faltung des Mnleküls längs der in Abb. 2 eingezeichneten Achse A (ähnliche Effekte werden beim Phenanthren diskutiert, bei dem die Wechselwirkung der Protonen am C-4 und C-5 das Molekül deformieren solll ${ }^{11}$ )).

Die Faltung des Moleküls bedingte in IX einen Rückgang des Ringstromeffektes und eine Verringerung der durch den Stickstoff hervorgerufenen paramagnetischen Verschiebung. Für die Signallage der Protonen, die der Faltungsachse naheliegen, resultierte daraus — im Vergleich zu den Signalen des Norcoralyns - eine Verschiebung zu höheren Feldern. Nur die an der Peripherie des Moleküls liegenden Protonen H-4 und H-9 werden nicht von dieser Verschiebung betroffen. Das Ausmaß der diskutierten Faltung ist jedoch nicht so stark, daß eine Veränderung im Chromophor einträte. Die UV-Spektren der besprochenen Coralyn-Derivate (Abb. 3) zeigen in allen drei Fällen einen sehr ähnlichen Kurvenverlauf.

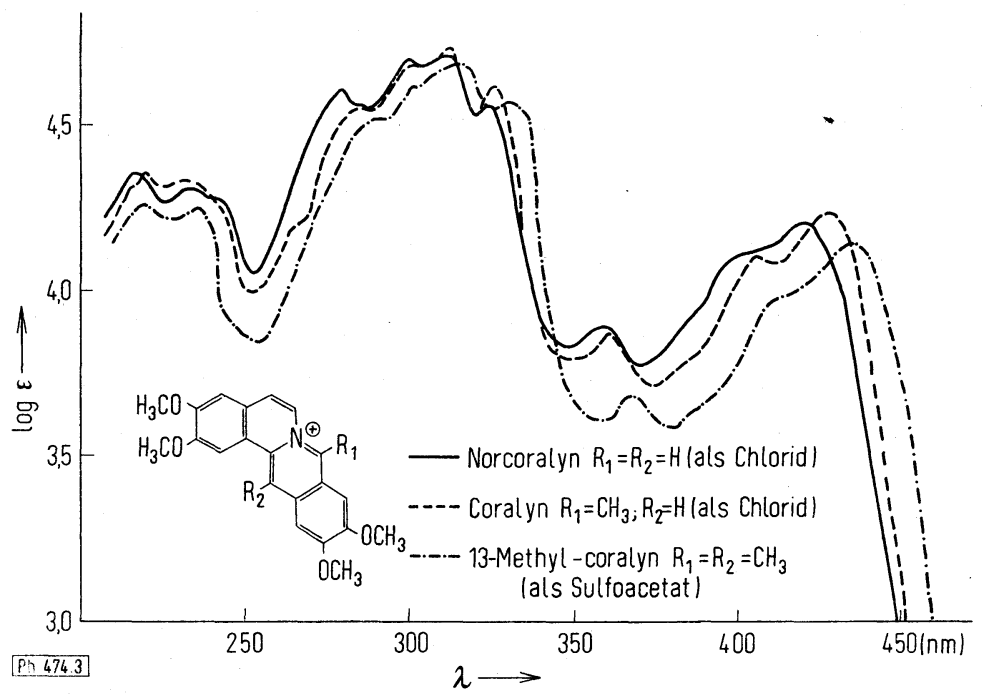

Abb. 3

Durch die auxochrome Wirkung der Methylgruppen wird in den methylsubstituierten Coralyn-Abkömmlingen die Lage der Maxima bathochrom verschoben. Eine starke Faltung müßte jedoch einen entgegengesetzten Effekt bewirken.

In diesem Zusammenhang interessierte uns die Struktur des Sulfoacetat-Anions im Coralyn-Salz. Die Tatsache, daß zur Coralyn-Bildung Acylkationen auf Benzylisochinoline einwirken müssen, spricht für die Struktur eines gemischten Anhydrides. Andererseits ist bekannt, daß Acetylschwefelsäure (a) nur unter $0^{\circ}$ beständig ist ${ }^{12}$ ). Bei der präparativen Gewinnung des Coralyn-sulfoacetates arbeitet man auf dem siedenden Wasserbad ${ }^{1}$ ).

11) C. Reid, J. Mol. Spec. 1, 18 (1957); zitiert nach $H$. Suhr, Anwendung der kernmagnetischen Resonanz in der organischen Chemie, Springer-Verlag, Berlin 1965, S. 181/182.

12) DRP 275846, C. 1914 II, 366. 


$$
\mathrm{H}_{3} \mathrm{C}-\mathrm{CO}-\mathrm{O}-\mathrm{SO}_{3} \mathrm{H}
$$

(a)

$$
\mathrm{HOOC}-\mathrm{CH}_{2}-\mathrm{SO}_{3} \mathrm{H}
$$

(b)

Während im NMR-Spektrum des Coralyn-sulfoacetates der Methoxylprotonen-Bereich ein Integral von 14 statt 12 Protonen ausweist, werden beim Coralyn-chlorid exakt 12 Protonen gemessen. Eine Verschiebung der $\mathrm{CH}_{2}$-Protonen des Anions in den Bereich der Methoxylprotonen ( $\tau=5,7 \mathrm{bis} 5,9 \mathrm{ppm}$ ) spricht für die Struktur der Sulfoessigsäure (b), deren Anion im Coralyn ( $\mathrm{I}, \mathrm{R}^{\prime}=\mathrm{CH}_{3}$ )-Salz vorliegt.

Dem Land Niedersachsen sind wir für die Förderung dieser Arbeit durch Forschungsmittel zu Dank verpflichtet.

\section{Beschreibung der Versuche}

Coralyn (I, $\mathrm{R}^{\prime}=\mathrm{CH}_{3}$ ) und $6^{\prime}$-Acetyl-papaverin (III) wurden nach ${ }^{1}$ ) hergestellt.

\section{Oxydativer Abbau von III zu IV}

$\mathrm{Zu}$ einer Lösung von $9 \mathrm{~g} \mathrm{NaOH}$ in $50 \mathrm{ml}$ Wasser wurden unter Rühren und Eiskühlung $9,6 \mathrm{~g}$ Brom so langsam zugetropft, daß die Temp. nicht über $10^{\circ}$ anstieg. In die auf $0^{\circ}$ abgekühlte Hypobromit-Lösung wurde die gesättigte Lösung von $7 \mathrm{~g}$ III in abs. Dioxan unter Rühren unterhalb $10^{\circ}$ eingetropft. Anschließend wurde $1 \mathrm{Std}$. bei Raumtemp. gerührt. Durch dreimaliges Ausschütteln mit Äther wurde Bromoform entfernt, die alkalische Lösung wurde zur Zers. überschüssigen Hypobromits mit $2 \mathrm{~g}$ Natriumpyrosulfit in $30 \mathrm{ml}$ Wasser versetzt und dann mit konz. Salzsäure stark angesäuert. Es schied sich ein Kristallbrei von IV-Hydrochlorid ab, das aus salzsaurem Wasser mit Kohlezusatz umkristallisiert wurde. Schmp. 246-248 ${ }^{\circ}$, Ausbeute an analysenreinem Produkt 58\% d. Th.

$$
\begin{array}{lll}
\mathrm{C}_{21} \mathrm{H}_{22} \mathrm{NO}_{6} \mathrm{Cl} \cdot \mathrm{H}_{2} \mathrm{O}(437,8) & \text { Ber.: C 57,60 } & \mathrm{H} \mathrm{5,54} \\
& \text { Gef.: C 57,71 } & \mathrm{H} \mathrm{5,59}
\end{array}
$$

\section{IV-Methylester-Hydrochlorid}

$4 \mathrm{~g}$ IV-Hydrochlorid wurden in $50 \mathrm{ml}$ abs. Methanol gelöst. In die Lösung wurde $20 \mathrm{Min}$. HCl-Gas eingeleitet, danach 2 Std. unter Rückfluß gekocht. Beim Erkalten fiel ein gelber Kristallbrei aus, der aus Methanol/wäßriger $\mathrm{HCl}$ umkristallisiert wurde. Schmp. $160^{\circ}$ (Zers), Ausbeute: $\mathbf{7 5} \%$ d. Th.

$$
\begin{array}{lll}
\mathrm{C}_{22} \mathrm{H}_{24} \mathrm{NO}_{6} \mathrm{Cl} \cdot \mathrm{H}_{2} \mathrm{O}(451,9) & \text { Ber.: } \mathrm{C} \mathrm{58,47} & \mathrm{H} 5,80 \\
& \text { Gef.: C 58,70 } & \text { H 5,53 }
\end{array}
$$

\section{IV-Methylester}

$3 \mathrm{~g}$ IV-Methylester-Hydrochlorid wurden in siedendem Wasser gelöst. Nach dem Erkalten wurde die Lösung mit Ammoniak auf $\mathrm{pH}$ 8-9 eingestellt und mit Chloroform ausgeschüttelt. Nach dem Trocknen und Einengen der Chloroform-Lösung wurde mit dem 3fachen Vol. Äther versetzt. Der Ester fiel kristallin aus und wurde aus Methanol umkristallisiert. Schmp. $256^{\circ}$, Ausbeute: $76 \%$ d. Th.

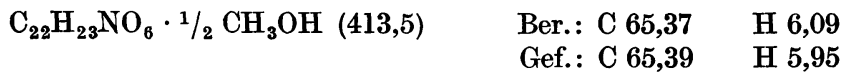

Im 100-MHz-NMR-Spektrum (TMS als innerer Standard, $\mathrm{CDCl}_{3}$ als Lösungsmittel) trat bei $\tau=6,58 \mathrm{ppm}$ das Methanol-Singulett mit einem Integral von 1,5 Protonen auf. 


\section{Reduktion von IV-Methylester zu V}

$1,5 \mathrm{~g}$ Ester wurden in abs. Tetrahydrofuran gelöst und zu einer Suspension von $\mathbf{0 , 9} \mathrm{g}$ $\mathrm{LiAlH}_{4}$ in Äther unter Rühren so zugetropft, daß die Reaktionsmischung schwach siedete. Anschließend wurde noch 15 Min. gerührt, überschüssiges Reduktionsmittel mit Wasser zersetzt, die Ätherphase abgegossen und der Niederschlag mehrmals mit Methylenchlorid extrahiert. Die vereinigten organischen Lösungen wurden über Natriumsulfat getrocknet und bei $40^{\circ}$ Wasserbadtemp. i. Vak. zur Trockne eingeengt. Ein gelbbrauner Niederschlag blieb zurück, Umkristallisation aus Methanol gab weiße Kristalle, Schmp. $172^{\circ}$. Ausbeute: $67 \%$ d. Th.

$$
\begin{array}{lll}
\mathrm{C}_{21} \mathrm{H}_{23} \mathrm{NO}_{5}(369,4) & \text { Ber.: C 68,28 } & \text { H 6,28 } \\
& \text { Gef.: C 68,36 } & \text { H 6,38 }
\end{array}
$$

\section{Norcoralynchlorid (I, $R^{\prime}=H$ ) aus $V$}

$0,5 \mathrm{~g} \mathrm{~V}$ wurden in $20 \mathrm{ml} 50$ proz. Essigsäure gelöst und mit einer Lösung von $0,4 \mathrm{~g}$ Natriumdichromat in $5 \mathrm{ml}$ 50proz. Essigsäure versetzt. Beim Stehen bei Raumtemp. bildete sich nach $30 \mathrm{Min}$. ein feinkristalliner Niederschlag. Nach vierstündigem Stehen wurde abgenutscht, mit wenig 50proz. Essigsäure gewaschen und mehrmals aus salzsaurem Methanol umkristallisiert. Ausbeute: 59\% d. Th., Schmp. 236-240 (Z), Lit. $\left.{ }^{4}\right): 240-242^{\circ}$.

\section{Norcoralyn-Hydrogenchromat}

Ansatz wie bei Norcoralynchlorid, beim Umkristallisieren aus Methanol wurde jedoch keine Salzsäure hinzugesetzt. Dieses Salz schmilzt nicht unter $300^{\circ}$.

$$
\begin{array}{lll}
\mathrm{C}_{21} \mathrm{H}_{20} \mathrm{NO}_{4} \cdot \mathrm{HCrO}_{4}(467,4) & \text { Ber.: C 53,97 } & \mathrm{H} \mathrm{4,53} \\
& \text { Gef.: C 54,36 } & \mathrm{H} \mathrm{4,70}
\end{array}
$$

\section{Oxydation von $\mathrm{V}$ mit aktivem $\mathrm{MnO}_{2}$}

$0,2 \mathrm{~g} \mathrm{~V}$ wurden in $10 \mathrm{ml}$ Chloroform gelöst, mit $1,5 \mathrm{~g}$ aktivem $\mathrm{MnO}_{2}$ versetzt und bei Raumtemp. 6 Std. gerührt. Der Braunstein wurde abgenutscht und anschließend im Durchlaufextraktor mit Chloroform extrahiert. Die vereinigten Chloroformlösungen wurden i. Vak. zur Trockne eingeengt. Das anfallende Öl kristallisierte nach kurzer Zeit, die Kristalle wurden aus Methanol umkristallisiert. Gelbe Nadeln. Schmp. 258-259 ${ }^{\circ}$.

$$
\begin{array}{lll}
\mathrm{C}_{21} \mathrm{H}_{19} \mathrm{NO}_{5}(365,4) & \text { Ber.: C 69,03 } & \text { H 5,24 } \\
& \text { Gef.: C 69,45 } & \text { H 5,73 }
\end{array}
$$

\section{Synthese von IX}

9-Methylenpapaverin wurde nach ${ }^{13}$ ), 9-Methylpapaverin (VIII) in Anlehnung an ${ }^{14}$ ) hergestellt: $5 \mathrm{~g}$ Methylenpapaverin wurden unter Erwärmen in $13 \mathrm{ml} 50 \mathrm{proz}$. Essigsäure gelöst. Danach gaben wir $52 \mathrm{ml}$ Wasser und $5 \mathrm{~g}$ Pd-Kohle (5proz.) hinzu und hydrierten unter heftigem Rühren. Berechnet waren $389 \mathrm{ml} \mathrm{H}_{2}$, aufgenommen wurden $386 \mathrm{ml}$. Das filtrierte Reaktionsgemisch wurde mit Natriumcarbonat alkalisiert, VIII schied sich ölig ab, kristallisierte aber bald. Die alkalische Lösung wurde mit Methylenchlorid extrahiert, das Lösungsmittel abdestilliert und der Rüekstand in Äthanol aufgenommen. Auch das kristalline Rohprodukt wurde aus Äthanol umkristallisiert. Schmp. $154^{\circ}$ (Lit.: $154^{\circ}{ }^{14}$ )). Ausbeute: $80 \%$ d. Th.

$\left.{ }^{13}\right)$ E. Späth und N. Polgar, Ber. dtsch. chem. Ges. 62, 1027 (1929).

14) E. Späth und E. Kruta, Ber. dtsch. chem. Ges. 59, 2787 (1926). 


\section{Umsetzung von VIII zu IX-Sulfoacetat}

$0,4 \mathrm{ml}$ konz. Schwefelsäure und $2 \mathrm{ml}$ Acetanhydrid wurden zu Sulfoessigsäure umgesetzt ${ }^{1}$ ). Daraufhin wurden 0,5 g VIII hinzugegeben, und das Reaktionsgemisch wurde 1 Std. im siedenden Wasserbad erhitzt. Beim Erkalten schieden sich leuchtend gelbe Kristalle ab. Um überschüssiges Acetanhydrid umzusetzen, wurde der Kristallbrei mit Methanol verrieben und nach 1 Std. abgesaugt. Die methanol. Lösung der Kristalle fluoreszierte gelbgrün. Aus Wasser umkristallisiert, schmolzen die Kristalle bei $\mathbf{1 7 3}^{\circ}(\mathrm{Z})$. Ausbeute: $\mathbf{5 5 \%}$ d. Th.

$$
\begin{array}{lll}
\mathrm{C}_{23} \mathrm{H}_{24} \mathrm{NO}_{4} \cdot \mathrm{C}_{2} \mathrm{H}_{3} \mathrm{SO}_{5} \cdot 2,5 \mathrm{H}_{2} \mathrm{O}(562,6) & \text { Ber.: C 53,37 } & \mathrm{H} 5,73 \\
& \text { Gef.: C } 53,24 & \mathrm{H} 5,98
\end{array}
$$

\section{3-Methyl-coralydin (X), Racemate I und II}

0,3 g IX-Sulfoacetat wurden in einer Mischung aus $18 \mathrm{ml}$ 5proz. Schwefelsäure, $9 \mathrm{ml}$ Eisessig und 1,5 ml wasserfreier Ameisensäure $5 \mathrm{Std}$. mit Zinkstaub reduziert. Nach dem Abkühlen wurde die Base wie üblich durch Fällung mit Ammoniak gewonnen; das DC der ätherischen Lösung (Kieselgel GF 254; Fließmittel: Benzol-Methanol-Aceton $6: 1: 3$ ) zeigte, daß zwei Dragendorff-positive Verbindungen vorhanden waren. Das Basengemisch wurde in Methanol aufgenommen, aus dem Racemat I $(\mathrm{Rf}=0,79)$ bevorzugt kristallisierte. Racemat I schmolz nach dreimaligem Umkristallisieren aus Methanol bei 169,5-170 .

$$
\begin{array}{lll}
\mathrm{C}_{23} \mathrm{H}_{29} \mathrm{NO}_{4}(383,5) & \text { Ber.: C 72,03 } & \mathrm{H} \mathrm{7,61} \\
& \text { Gef.: C 71,85 } & \text { H 7,58 }
\end{array}
$$

Racemat II $(\mathrm{Rf}=0,57)$ wurde durch Schichtchromatographie von Racemat I getrennt. Dazu wurden die Mutterlaugen des Racemates I eingeengt und auf $20 \times 20 \mathrm{~cm}$-Platten chromatographiert, die mit $80 \mathrm{ml}$ einer Suspension von $90 \mathrm{~g}$ Kieselgel PF 254 in $225 \mathrm{ml}$ Wasser beschichtet worden waren. Fließmittel: Benzol 60, Aceton 35, Methanol $5 \mathrm{v} / \mathrm{v}$. Nach zweimaligem Entwickeln wurde die Zone des Racemates II abgeschabt und mit Methanol eluiert. Racemat II schmolz nach dreimaligem Umkristallisieren aus Methanol bei $142-143,5^{\circ}$.

$$
\begin{array}{lll}
\mathrm{C}_{23} \mathrm{H}_{29} \mathrm{NO}_{4}(383,5) & \text { Ber.: C 72,03 } & \mathrm{H} 7,61 \\
& \text { Gef.: C 71,84 } & \text { H 7,55 }
\end{array}
$$

\section{Allgemeine Angaben}

Die Schmp. wurden im Kupferblock bestimmt und sind unkorrigiert angegeben. Die UV-Spektren wurden in methanolischer Lösung im Zeiss-Spektralphotometer PMQ II, die IR-Spektren im Beckman-Gerät IR 5 aufgenommen. Für die NMR-Spektren stand das Gerät HA 100 der Fa. Varian zur Verfügung.

Den Herren Prof. Dr. Awe und Prof. Dr. Perkampus danken wir verbindlich für ausführliche Diskussionen; Herrn Prof. Dr. Zinner sind wir für die IR-Spektren, Herrn Prof. Dr. Dr. h. c. Inhoffen für die NMR-Spektren zu Dank verpflichtet. Die Fa. E. Merck AG hat diese Arbeit dankenswerterweise durch Chemikalienspenden nachhaltig gefördert.

Anschriften: Priv.-Doz. Dr. W. Wiegrebe, 33 Braunschweig, Pockelsstr. 4.

Dr. U. Krüger, 3301 Stöckheim b. Braunschweig, Institut für Molekulare Biologie, Biochemie und Biophysik.

(c) Verlag Chemie GmbH, Weinheim/Bergstr., 1968

Bei der Wiedergabe von Gebrauchsnamen, Handelsnamen, Warenbezeichnungen u. dgl. handelt es sich häufig um gesetzlich geschützte eingetragene Warenzeichen, auch wenn sie nicht als solche mit ${ }^{\circledR}$ gekennzeichnet sind. 